\title{
LE RAPPORT À L'HISTOIRE DANS L'IMAGINAIRE DIASPORIQUE DES ROMANCIERS HAÏTIENS DU QUÉBEC
}

\author{
Józef KWATERKO \\ Institut d'études romanes \\ Université de Varsovie
}

\begin{abstract}
En): This paper is devoted to different ways in which Haitian novelists inscribe history in their narratives. Three imaginary approaches of space and time are to be distinguished: exile, autofiction, and post-exile. Socio-critical readings of Haitian novels will reveal the framing of individual and collective history, the historicity of the text itself, and in particular the historical representations embedded in the social discourse, its system of values and its ideology.

Résumé (Fr) : Cette contribution se propose de cerner différentes modalités de l'inscription de l'histoire dans la fiction des romanciers haïtiens du Québec selon trois approches imaginaires de l'espace-temps représenté : « exilique», autofictive et "postexilique ». Moyennant une lecture sociocritique du roman, l'on sera attentif en particulier au rapport entre la mise en scène de l'histoire (collective et individuelle) et l'historicité textuelle (le travail du texte sur des représentations historiques inscrites dans le discours social, ses cadres axiologiques et idéologiques).
\end{abstract}

Keywords (En): Haiti; Québec; novel; individual history; collective history

Mots-clés (Fr) : Haïti ; Québec ; roman ; histoire individuelle ; histoire collective

Dans Haïti. Une traversée littéraire, ouvrage publié en 2010, au lendemain du tremblement de terre par Louis-Philippe Dalembert et Lyonel Trouillot, on peut lire un jugement d'ensemble sur le rapport à l'histoire dans la littérature haïtienne :

Comment, au bout du compte, les œuvres littéraires donnent-elles à lire l'histoire du pays ? - D'abord, on y trouve, il faut le dire, la volonté didactique de dénoncer, sur le plan local, les avatars de l'Histoire et de faire connaître le pays à un lectorat étranger. Ces ouvrages, au fond, donnent à lire une Histoire omniprésente, totale, voire totalitaire, une immense machine collective qui, à travers ses bégaiements successifs, broie, annihile tout destin individuel. L'histoire s'inscrit dès lors, selon le mot d'André Malraux, comme destin. Elle vient à la rencontre des personnages, sans que ceux-ci puissent s'en protéger. Mais, dans le cas de la nation haïtienne, née sur les ruines de la plantation coloniale, plus qu'un destin, elle se fait identité. Dès lors, chaque événement majeur constitue une composante, mieux, une étape dans la construction de cette identité, comme si le peuple ne savait se dire ou se définir autrement qu'à travers les grands moments souvent tragiques de son histoire (DALEMBERT et TROUILLOT, $2010: 28-30$ ).

On pourrait se demander comment, face à ce déterminisme lourd, les écrivains haïtiens du Québec problématisent le rapport à l'histoire en tant qu'enjeu identitaire. Il semble que, pour cerner les modalités de l'inscription de l'histoire dans leur fiction, l'on puisse distinguer trois approches imaginaires de l'Histoire qui ne sont pas étroitement successives et peuvent se superposer : 
- une approche " exilique », rivée à la mémoire de la persécution duvaliériste, qui privilégie une esthétique de la dégradation et montre le Québec en contrepoint comme lieu de malaise identitaire ;

- une approche autofictive, qui privilégie « le retour à l'enfance créole » en vue de rétablir les liens entre la mémoire familiale et la mémoire culturelle de l'écrivain en diaspora ;

- une approche "postexilique », observable à partir de la fin des années 1990, où le sentiment de l' " ex-île » (DES Rosiers, 1996 : 4) permet de parler du Québec comme espace-temps «identitaire », vécu à travers l'historicité de ses propres métamorphoses

\section{Mémoire de la persécution}

Il convient de rappeler que certains auteurs haïtiens de la première vague d'immigration au Québec, celle des années 1960 et 1970, comme Anthony Phelps, Gérard Étienne ou Émile Ollivier, ont personnellement connu les persécutions de François Duvalier. Exilés au Québec, ils cherchent à exprimer le cauchemar qu'ils ont vécu en Haïti, obsédés à la fois par ce qu'ils doivent laisser au pays natal, mais aussi par le sentiment de l'impossibilité de vivre une vie normale, sous l'apparence du bonheur quotidien retrouvé dans leur patrie adoptive. Leur écriture « exilique » est marquée plutôt par un "devoir de mémoire » et animée par la passion du témoignage et par une mémoire immédiate. Étant donné que l'histoire d'Haïti (la mémoire des racines africaines ainsi que l'attachement à la terre natale, dépositaire de traditions culturelles et spirituelles) avait été érigée en idéologie par un état totalitaire, le récit diasporique haïtien va être le plus souvent fondé sur le souvenir personnel des cruautés duvaliéristes. Encombré d'événements traumatiques et d'images du désastre collectif haïtien, ce récit aura pour objectif premier de révéler et dénoncer les crimes. La mémoire de la terre-mère meurtrie étant encore très vive, viscérale, les écrivains exilés haïtiens de cette période vont plutôt privilégier des récits "interventionnistes ", là où le chevauchement de fiction et de témoignage court-circuite toute narration nostalgique ou compensatoire.

On le voit bien dans Moins l'infini, roman d'Athony Phelps publié au Québec en 1973, où le souvenir lancinant des violences subies demeure irréductible, la vengeance sur le dictateur étant le repère majeur de l'intrigue: «L'éliminer. L'effacer, le tuer par strangulation [...] L'enterrer jusqu'au cou, puis enduire sa tête de miel et d'épice et le laisser à la merci des fourmis et des rats qui lui dévoreront ses lèvres épaisses, ses joues d'engraissé, ses yeux globuleux, son front buté, ses petites oreilles, jusqu'à ses lunettes » (PHELPS, 1973: 40).

Dans cette mémoire rivée à la douleur de l'oppression vécue, le présent domine. Dans La mémoire en colin-maillard, roman publié par Phelps trois ans plus tard, en 1976, ce temps se donne à lire comme le seul approprié à une action dominée par le désir de vengeance et fantasmée comme imminente :

A travers les branches de mon muscadier qui tressaillent au moindre souffle venu de la montagne proche, je fais le guet. Replié comme un chat dans mon vieux fauteuil, je surveille les alentours [...] Je suis le guetteur montant la garde de la résignation dans mon poste de feuillage [...] je reconnais au bruit, à quatre 
rues d'ici, la marque des voitures qui passent. Au moindre crissement insolite, mes oreilles se dressent, mes sens sont alertés. J'ai appris à distinguer les pas des inoffensifs de celui des bourreaux. Ils ne m'auront plus par surprise. J'attends, je surveille, je les domine de mon balcon. Je les vois aller et venir. Se déplacer comme des rats dans la rue. Pan ! ... une balle en plein front ! La semaine dernière, j'en ai abattu quatre alors qu'ils perquisitionnaient chez les Thibaud (PHELPS, $1976: 22-25)$.

Il en va de même dans Le Nègre crucifié de Gérard Étienne, roman publié en 1974 et dominé par les représentations de la violence dans un univers concentrationnaire, peuplé de zombies symbolisant les effets de la dépossession collective. Il faut observer que dans cette série de romans d'exil, le Québec se profile seulement dans le récit comme le lieu d'où l'on parle, le lieu d'énonciation qui libère la parole, sans toutefois pouvoir être représenté dans ses composantes spatiotemporelles. Une écriture militante, mobilisée par la volonté de dénonciation des crimes, ne peut avoir d'objet qu'Haïti, le plus souvent Port-au-Prince, lieu de honte, la ville où « les nègres coupent la gorge des nègres d'Haïti » (ÉTIENNE, 1974 : 110).

Lorsqu'en 2000 dans son récit autobiographique, Le cri des oiseaux fous, Dany Lafferière parlera de son départ précipité du pays natal, pourchassé par les sbires de Baby Doc (Jean-Claude Duvalier), il n'utilisera pas non plus un plan historique, mais un plan politico-mémoriel avec sa manière de parler au présent, se mettant ainsi dans une posture symétrique à celle du désastre haïtien qu'il dénonce :

Je me sens diablement présent dans cette ville maudite. J'ai conscience d'être, au mois pour cette nuit, l'élément pivotant de la ville [...] Partout on crie, on pleure, on hurle. Je marche tranquillement au centre du tumulte [...] Les portes des maisons sont déjà fermées. A l'intérieur, les femmes prient, agenouillées devant la Vierge, l'implorant de protéger leur fils. Les fils sont dans les rues. Les pères sont morts, ou en prison, ou en exil ou planqués sous les lits. Les femmes continuent à prier. L'effroyable défaite des pères. Papa Doc a bien pris soin d'éliminer ou d'humilier les pères en présence de leurs fils, leur enlevant toute autorité pour permettre à son fils à lui, Baby Doc, de monter sur le trône (LAFERRIÈRE, 2000 : 125).

Pour Émile Ollivier, parler du passé haïtien à partir de l'exil, c'est aussi parler d'un désastre collectif - un fardeau lourd à porter qui contient un réservoir inépuisable de douleurs, de violence et d'oppressions impossibles à oblitérer. Comme il va l'avouer une année avant sa mort: "Le passé, on le traîne avec soi, comme la poussière que l'on traîne avec la semelle de ses chaussures. Il n'y a pas de doute, on n'échappe pas à son passé. Le passé nous hante. Le passé nous poursuit. Le passé déguise parfois la nouvelle réalité » (MONTPETIT : 2001). Il est significatif que dans Mère solitude, roman publié en 1983, Émile Ollivier puise dans le gouffre de l'histoire haïtienne pour retracer, sur un mode chaotique, fragmentaire, la vie d'une famille, les Morelli, marquée par les crimes et la haine qu'elle subit depuis plusieurs générations. On y retrouve le portrait d'une ville, Trou-Bordet, qui ressemble à Port-au-Prince, ville morte, lieu de décomposition et d'effondrement physique et moral dont le narrateur décrit les miasmes sur un mode apocalyptique : 
Que puis-je dire de ce pays ? Que puis-je dire de cette ville ? [...] Je suis né et j'ai grandi dans cette ville vomie par la mer, coincée par la montagne. Que saisje de la montagne, sinon son dos de rat pelé, galeux, sa face ravinée ? [...] Perdu dans les abysses de mes paysages intérieurs, je me suis assigné à moi-même cette exploration muette de mon passé et celui de mon pays. [...] Je traverse d'un pas pressé la ville, règne de l'insensé. [...] On n'aura jamais fini de la décrire. Ville de la basilique unique, du grand gibet et de la boue ! Cette ville dégouline vers la mer comme un abcès. Ville de l'incandescence comme feu d'épines en plein vent, paroxysme jamais atteint de merveilles et de terreurs ! Ils t'ont appelée TrouBordet, mais tu es également Trou-aux-Vices, Trou-aux-Assasins, Trou-auxCrimes. Ville de sang et d'ordures ! Ville aux aguets ! Ville de bitume et de trou ! On n'aura jamais fini de te décrire (OLLIVIER, 1983 : 27-28).

Cette «esthétique de la dégradation» (LUCAS, 2002, 191-211), dont la prégnance marque aussi puissamment la littérature haïtienne « du dedans » (celle qui s'écrit en Haïti), prend chez les romanciers haïtiens exilés au Québec des formes variées. Par exemple, dans Le livre d'Emma de Marie-Célie Agnant, publié en 2001, la réécriture de l'Histoire prend la forme du récit des origines qui progresse par bribes, par des souvenirs hachés et des propos incohérents d'Emma Baratte, écrouée pour infanticide dans un hôpital psychiatrique de Montréal et refusant de communiquer en français avec le personnel soignant. Enfermée dans son mutisme, elle va finir par communiquer en créole à Flore, son interprète et narratrice, les zones obscures de son enfance, et au-delà, le passé africain de son peuple contraint à l'esclavage, au " ventre du bateau négrier et l'antre de la Plantation » (AGNANT, 2001 : 133). Cette inscription d'une histoire collective dans le récit d'une vie individuelle deviendra pour Emma une façon de colmater ses déchirements et son déracinement psychique et de donner une unité à son moi, à sa vie et à son corps de « négresse ».

\section{Retours à l'enfance}

Dans la démarche autofictive des romanciers québécois d'origine haïtienne, le " devoir de mémoire », marqué par une mémoire fondamentale, qui refuse de faire le deuil de la déprise historique d'Haïti, cède la place à un travail de mémoire qui met en jeu une mémoire affective et fonctionne par associations libres à la manière de la « madeleine de Proust ». La vie en exil, la distance, autorise une prise de recul ; à une mémoire patrimoniale, saturée du passé collectif, se substituent d'autres formes mémorielles qui permettront de colmater les lignes de faille. Comme l'avoue Émile Ollivier : « Demeurant écartelé entre ma terre d'origine et mon lieu d'arrivée, je vivais un malaise permanent. Jusqu'au moment où, récusant la part de malheur et de souffrances liée au déplacement, j'ai composé avec toutes les cartes que me remettait la vie. Cette prise de distance a entraîné une profonde révision de moimême » (OLLIVIER, $2001: 23$ ).

Désormais, la ligne entre mémoire et histoire se fait plus fragile, plus mouvante. Vient le temps où préserver son identité haïtienne consiste à instaurer, sur un mode individuel et moins idéologique, un nouveau rapport dialogique avec soi même et avec le pays délaissé. Ce qui fait le retour dans le discours, ce sont la mise en scène de l'enfance et la mémoire familiale, intime et jusqu'alors refoulée. Les romans de 
Dany Laferrière et d'Émile Ollivier décomposent les grands récits du passé, les fragmentent et proposent des reconfigurations narratives qui se tissent et détissent à travers un dédale de souvenirs disjoints, dispersés, entremêlés ou alternés, hors de toute quête totalisatrice de sens. On le voit bien dans l'incipit de L'odeur du café de Dany Laferrière, récit autofictif publié en 1991, où le besoin de renouer avec les liens familiaux, de coller au hic et nunc haïtien, de se situer enfin «chez soi », comme si on ne l'a jamais quitté, est de l'ordre du fantasme de continuité longtemps refoulé :

L'Été 63. J'ai passé mon enfance à Petit-Goâve, à quelques kilomètres de Port-au-Prince. Si vous prenez la Nationale Sud, c'est un peu après le terrible morne Tapion. Laissez rouler votre camion (on voyage en camion, bien sûr) jusqu'aux casernes (jaune feu), tournez tranquillement à gauche, une légère pente à grimper, et essayez de vous arrêter au 8 de la rue Lamarre. Il est fort possible que vous voyiez, assise sur la galerie, une vieille dame au visage serein et souriant à côté d'un petit garçon de dix ans. La vieille dame, c'est ma grand-mère. Il faut l'appeler Da. Da tout court. L'enfant, c'est moi. Et c'est l'été 63. (LAFERRIÈRE, 1991: 13).

Toutefois, aborder l'Histoire dans le récit autofictif repose souvent, comme le formule Dominique Viart, sur une «reconstruction hésitante et inquiète d'expériences partielles »(VIART, 2005, 125-126). Dans Mille eaux, publié en 1999, où Émile Ollivier revient sur son enfance haïtienne, lieu par excellence de la mémoire intime, la pulsion du retour échappe à toute cohérence des réminiscences, malgré le caractère autobiographique du récit. Même si l'auteur se dit " ethnologue » de soi-même, "parti à la recherche des images fondatrices " (OLLIVIER, 1999 : 78), son retour au passé y signifie avant tout un retour sur soi : une façon tâtonnante, faite d'une série infinie de ruptures, de ressusciter les secrets d'un destin individuel. En regard de cette autofiction, on pourrait parler, à la suite de Réal Ouellet, d'une écriture de « réenracinement 》 (OUELLET, $2000: 283$ ), d'une reconstruction du passé qui ne sépare pas de la scène historique haïtienne, mais qui l'installe dans l'infra-ordinaire de la petite histoire, avec son imaginaire de sensations, de filiations et de langues de tous les lieux parcourus :

Alors que le monde, au début des années cinquante, vivait en pleine révolution industrielle, avec ses usines, ses sirènes, ses travailleurs à la chaîne, moi, j'étais installé à Martissant, cette cité hors du temps. [...] Pendant que je regardais passer les marines américaines en permission, sensibles aux charmes des éphèbes et des putes dominicaines, des bordels avoisinants montaient des voix aux accents étrangers, voix latino-américaines, rauques, basses, modulées, voix roucoulantes de querido mio par-ci, de aïe mi corazon par-là, voix qui me pénétraient et me sortaient de mes peurs, voix qui retentissaient en moi comme des hennissements de chevaux sauvages galopant fous à travers les champs. La musique de ces noms que j'ai retrouvés - éclairés par des néons - beaucoup plus tard, à ma grande surprise, à Mexico, boulevard de los Insurgentes, Plaza de la Passeo Reforma : La Sonora Matansera, Celia Cruz, Perez Prado, Daniel Santos, caracolant au sommet des hit-parades. Des airs de meringue, de danson, de chacha-cha s'échappaient des juke-box. Jeune pousse, je poussais, bourgeon vert, graine bien membrée, sous les voluptés du perpétuel été, guettant les amours 
éphémères des papillons de la Saint-Jean, zyeutant les filles impériales aux yeux bridés de Shanghai, le seul teinturier à deux lieues à la ronde, ces filles, variations infinies de mélange de races et de cultures. Parlions-nous de métissage, déjà à ce moment-là ? (OLLIVIER, 1999: 101-102).

\section{Montréal : vers le « repaysement »}

Une troisième modalité d'inscription de l'histoire, visible à partir de la fin des années 1990, aurait trait à ce qu'on peut appeler une position "post-exilique», lorsque la fiction diasporique des écrivains haïtiens va subir une remodélisation esthétique en confrontation avec l' " ailleurs » québécois, c'est-à-dire avec le présent de l'exil. On pourrait emprunter ici le concept de Joël Des Rosiers qui, dans Théories caraïbes. Poétique du déracinement, parle de «l'effet d'ex-île » (DES RosIERS, 1996 : 3) Ce concept traduit un nouveau positionnement identitaire de l'écrivain haïtien, installé en diaspora québécoise à l'âge précoce (enfant ou adolescent) et libéré des angoisses de l'exil et du sentiment de l'écartèlement entre Haïti et le Québec. On peut dire que la mémoire des origines reste pour lui plus fictive que réelle, ce qui l'autorise à déployer des modalités d'écriture qui mettent à distance la nostalgie du pays natal ou encore à travailler ce manque comme un fantasme (voir DES Rosiers, 1996 : 10-15). On pourra penser ici à Stanley Péan, né à Port-auPrince en 1966 et ayant grandi au Québec, qui, en 1988, dans un des ses récits d'exils intitulé « Le syndrome Kafka », évoque le fantasme de l'origine comme résultat de la distanciation mémorielle:

[...] tu t'absentes de ta patrie trop longtemps, la métamorphose s'opère, l'exil fait de toi un intrus, un étranger partout même chez toi [...]. Tu en viens à oublier [...] ton créole par bribes. Oublier le visage de ton père. Il ne te reste plus d'Haïti qu'un vague parfum de cannelle, d'anis, de mangue que tu ne distingues plus très bien [...]. Une poignée de lettres que tu ne prends même plus le temps de lire. Une famille-fantôme à laquelle tu envoies un chèque une fois de temps à autre et de tes nouvelles encore moins souvent. Et une image de ton pays pas tout à fait exacte, filtrée par ta propre mémoire... (PÉAN, 1988 : 116-117).

Cette amnésie partielle permettra à Péan de situer l'action de son thriller Zombi Blues (1996) à Montréal et d'aborder, en arrière plan des meurtres perpétrés dans la communauté des immigrés haïtiens par un ancien ministre «macoute », arrivé en plein festival de Jazz à Montréal, la question de l'historicité de la métropole québécoise vue à travers la mobilité sociale des différentes communautés culturelles :

$\mathrm{Au}$ fil des dernières trente et quelques années, le paysage de ce quartier du nord de la métropole s'est considérablement modifié. Si bien que là où, auparavant, on apercevait des enseignes de comptoirs à hot dogs ou à patates frites ont proliféré des écriteaux lumineux aux couleurs vives annonçant des salons de coiffure « afro », boites de nuit antillaises, marchés de fruits et légumes dits « exotiques ». Les Québécois de souche, comme on les appelle maintenant, côtoient les ressortissants haïtiens depuis si longtemps que certains dépanneurs du coin offrent désormais du Cola-Champagne et autres douceurs des Tropiques. 
Certes, cette cohabitation n'est pas toujours aisée, comme en témoignent quelques graffitis haineux (Négro : mange-marde!), que l'on peut parfois lire sur le flanc des conteneurs à déchets, ou les occasionnelles rixes entre gangs de jeunes Noirs et skinheads. Mais malgré ces désagréments, moins fréquents que les médias les laissent entendre, Marie-Marthe estime son coin paisible, comparé à d'autres, et s'imagine difficilement vivre ailleurs dans Montréal (PÉAN 1999 : 41-42).

Ainsi, à travers la ville et les échanges migratoires, c'est tout le problème de la culture comme facteur d'intégration et d'assimilation dans le tissu urbain qu'il faut évoquer. On pourrait dès lors observer une mise en fiction du sentiment d'appartenance, une « reterritorialisation » de Montréal, si l'on peut dire ainsi par analogie au processus de réappropriation de la langue par lequel le sentiment identitaire retrouve un certain sens (voir BERTRAND, 2005 : 175-177).

S'emparer de l'historicité de la ville est aussi très visible dans le roman posthume d'Émile Ollivier, La Brûlerie, publié en 2004. En effet, Montréal s'offre ici d'emblée comme une ville identitaire. Le titre, La Brûlerie, est le nom du café situé sur le chemin de la Côte-de-Neiges près de l'Université de Montréal. Il renvoie à un espace familier où se tient le quartier général du " Ministère de la parole »-un groupe d'Haïtiens vieillissants qui discutent du présent et remontent le cours de leur vie en se remémorant leur passé. Or, on peut dire que leur véritable passé d'exil est surtout rattaché au passé du quartier Côte-de-Neige, à un rituel de flâneries et de rencontres qui dure déjà plus de trente-cinq ans. Dès l'incipit, la narration prend le ton d'un récit des origines qui rend manifeste la liaison consubstantielle, presque organique entre le quartier et le vécu du narrateur: "Je ressuscite depuis des décennies dans Côte-des-Neiges [...] Je connais tous les charmes et tous les pièges de ce quartier [...] J'ai entendu souffler l'Esprit de lieu, battre le rythme inhérent à cet espace » (OLLIVIER, $2004: 9$-10). Cet ancrage identitaire passe par l'évocation d'un archipel de café, de restaurants, de bistrots qui sont des véritables « lieux de mémoire ", entrelacés au passé-présent de ce quartier, devenu au fil des contacts une zone où s'exprime le mieux le cosmopolitisme montréalais. Dans son roman, Ollivier se montre non seulement un excellent cartographe de Montréal, de ses microterritoires à socialité affichée, mais aussi son parfait « sémiologue » ou, si l'on veut, historien culturel, qui capte par le filtre de l'imaginaire et d'un regard décalé, les métamorphoses et les mutations de cette ville que le narrateur définit comme « un lieu qui situe sans enclore » (OLLIVIER, 2004 : 164).

Se tourner vers Montréal pour en parler comme d'une ville identitaire, traduit assurément la situation " post-exilique » adoptée par l'écrivain haïtien en diaspora québécoise, une position de retrait qui tend à dédramatiser le poids de l'Histoire et le sens de l'exil, entendus comme déchirement ou folie, pour les transmuer en une forme de conscience supérieure laquelle permet de retrouver une liberté intérieure. Comme Ollivier l'avoue lui-même dans un commentaire métafictionnel dans $L a$ Brûlerie : «Quand les blessures se referment, quand elles ne font plus souffrir, elles deviennent des livres. Écrire est la meilleure façon d'échapper à la mort. Écrire, c'est dire, mais dire n'a d'intérêt que si c'est une tentative pour exprimer l'indicible » (OLLIVIER, 2004 : 104).

Ainsi, à le regarder depuis le Québec, le roman diasporique haïtien met en scène l'histoire comme un espace-temps labile, cumulatif d'expériences multiples - 
depuis des représentations anxiogènes, marquées par les images du désastre haïtien, le déchirement ou l'errance identitaire, jusqu'à des représentations plus apaisantes et dialogiques. Ces dernières, associées à la situation «postexilique » de l'écrivain et à Montréal, ville perçue désormais comme espace de vie, traduisent une forme de conscience supérieure qui permet de dédramatiser l'histoire et de garder une liberté intérieure.

\section{BIBLIOGRAPHIE}

Agnant Marie-Célie (2002), Le livre d'Emma, Montréal, Les éditions remueménage et Mémoire. BERTRAND Jean-Pierre (2005), «Territorialisation », in : BENIAMINO Michel, GAUVIN Lise (éds), Vocabulaire des études francophones. Les concepts de base, Limoges, Presses de l'Université de Limoges, 2005.

DAlembert Louis-Philippe, Trouillot Lyonel (2010), Haïti. Une traversée littéraire, Paris/Port-au-Prince, Presses nationales d'Haïti/Culturefrance éditions/Philippe Rey.

Des Rosiers Joël (1996), Théories caraïbes. Poétique du déracinement, Montréal, Triptyque.

ÉTIENNE Gérard (1974), Le nègre crucifié, Montréal : Éditions Francophones/Nouvelle Optique.

LAFERRIÈRE Dany (1991), L'odeur du café, Montréal, VLB éditeur.

LAFERRIĖRE Dany (2000), Le cri des oiseaux fous, Montréal, Lanctôt.

LUCAS Rafael (2002), L'esthétique de la dégradation dans la littérature haïtienne, Revue de littérature comparée, 302, Paris, Didier, p.191-211.

MONTPETIT Caroline (2001), Les promenades d'Émile Ollivier, entrevue, Le Devoir, 3 mars, p. D-1.

OLLIVIER Émile (1999), Mère solitude, Paris, Le Serpent à plumes.

OLLIVIER Émile (1999) Mille eaux, Paris, Gallimard (coll. « Haute enfance »).

OLLIVIER Émile (2001) Repérages, Montréal, Leméac.

OLLIVIER Émile (2004) La Brûlerie, Montréal, Boréal.

OUELLET Réal (2000) L'enfance dont en revient jamais : Le figuier enchanté de Marco Micone et Mille eaux d'Émile Ollivier, in : LEPAGE Yvan-G. et MAJOR Robert (dir.), Croire à l'écriture, Orléans (Ontario): Les Éditions David, p. 277289.

PÉAN Stanley (1988) La plage des songes et autres récits d'exil, Montréal: CIDHICA.

PÉAN Stanley (1999) Zombi Blues, Paris, J'ai lu (coll. « Ténèbres »).

PHELPS Anthony (1973) Moins l'infini, Montréal, CIDHICA.

PHELPS Anthony (1976) Mémoire en colin-maillard, Montréal : Nouvelle Optique. VIART Dominique (2005) Écrire l'Histoire, in : VIART Dominique, VERCIER Bruno (éds.) La littérature française au présent. Héritage, modernité, mutations, Paris, Bordas p. 125-206. 